\title{
Radio Station Background Noise Detection Based on Time- Frequency Domain Electromagnetic Spectrum
}

\author{
Fuzhai Wang, ${ }^{1}$ Zhenjia Chen $\mathbb{D}^{2},{ }^{2}$ Xuanfeng Chen, ${ }^{2}$ and Ting Chen ${ }^{2}$ \\ ${ }^{1}$ Transport Planning and Research Institute of MOT, Beijing, China \\ ${ }^{2}$ Hainan University, Haikou 570228, China \\ Correspondence should be addressed to Zhenjia Chen; zjchen@hainanu.edu.cn
}

Received 5 September 2021; Revised 24 September 2021; Accepted 28 September 2021; Published 15 October 2021

Academic Editor: Chi-Hua Chen

Copyright (C) 2021 Fuzhai Wang et al. This is an open access article distributed under the Creative Commons Attribution License, which permits unrestricted use, distribution, and reproduction in any medium, provided the original work is properly cited.

\begin{abstract}
The electromagnetic spectrum resource is one of the important national resources. It is a physical channel for wireless communication between ships and between ships and radio stations. Good communication quality must be guaranteed, so it is urgent to monitor and analyze the environmental background noise of the electromagnetic spectrum. The estimation of the radio frequency signal coverage in the target area during the monitoring process is of great significance to the study of electromagnetic spectrum resource management and control. This paper estimates the upper envelope and lower envelope of the background noise of the target frequency band based on the electromagnetic spectrum data in the time-frequency domain and combines the forward difference algorithm to estimate the background noise envelope curve. We set up fixed detection nodes and mobile detection nodes for specific construction areas and collect time-frequency spectrum data of electromagnetic spectrum in multiple locations. The instantaneous frequency spectrum and the collected data of a specific frequency point are compared, and it is difficult to judge whether there is a valid signal. This paper is based on the time-frequency domain electromagnetic spectrum data in the construction area of the project and estimates the background noise of the coast station frequency band in the current environment. It is based on the energy gradient estimation of the time-frequency domain spectrum, and the effective signal of the target frequency band is obtained and combines the noise envelope and the effective signal location to improve the estimation result of the background noise envelope. The experimental results show that the background noise estimation algorithm can reflect the changes in the noise floor of different target frequency bands.
\end{abstract}

\section{Introduction}

The electromagnetic spectrum resource is a nonrenewable national resource. Reasonable management and control of spectrum resources is the basic guarantee for wireless communication services and security. The safety management of maritime and ship traffic is particularly important, and maritime communication services are inseparable from the support of electromagnetic spectrum resources. In order to ensure the safety of ship traffic and meet the everincreasing demand for maritime communications, maritime radio plays a very critical role. The wireless walkie-talkies that carry out wireless communication between the ship on the sea and the shore are called marine wireless walkietalkies. It is also called a coast station. The background noise detection of the radio station is to ensure the communica- tion quality between the receiving station and the sending station. We need to detect the background noise in the area near the receiving station to find the interference source of the radio channel. We collect spectrum data on the coast and estimate the environmental background noise. The estimated results play a vital role in studying how to improve the communication quality in the sea and on the shore and can effectively improve the safety of maritime navigation.

\section{Related}

Coast station refers to a land station dedicated to ship transportation and shipping management services. Its main function is to timely detect the distress alarm from the ship and quickly transfer the alarm to the relevant search and rescue coordination center. It launches a shore-to-ship distress 
alert, and the search and rescue coordination center or search and rescue department can quickly obtain the alert information. It uses the radio interface between the land public communication network or the dedicated communication network and the ship station to play a switching role in the search and rescue coordination communication. Commonly used electromagnetic spectrum sensing methods include matched filter detection, cyclostationary feature detection, and energy detection (ED). Compared with the other two methods, the ED method has higher real-time performance and reliability. Due to its simplicity and applicability and its low calculation and implementation costs, ED constitutes the preferred method of spectrum sensing in cognitive radio [1]. In order to improve the detection probability, it uses a priori information related to the spectrum usage of the main user is used to enhance the spectrum sensing performance. [2] proposes a threshold setting method based on approximate analysis to achieve target detection probability or false alarm probability. [3] studied energy detectors based on dual thresholds for cooperative spectrum sensing mechanisms in cognitive radio networks. The setting method of the noise energy threshold determines the detection probability of the effective signal. [4] studied energy detectors based on dual thresholds for cooperative spectrum sensing mechanisms in cognitive radio networks. The author improves the detection probability of spectrum sensing by increasing the spatial dimension of spectrum detection. We propose a combined collaborative spectrum detection method to reduce the background noise of the overall detection system [5]. In large-scale cognitive radio networks, secondary users cannot share the public spectrum due to the limited coverage of primary users. [6] proposed a diffusion adaptive learning algorithm based on the correlation entropy cooperation strategy to realize public spectrum estimation.

In addition to energy detection methods, many scholars are also studying other spectrum sensing methods. [7] proposed a spectrum sensing scheme based on adaptive dual thresholds. The author compares it with the spectrum sensing technology based on the cyclostationary feature detector. At $8 \mathrm{~dB}$ signal-to-noise ratio (SNR), the detection probability of MED is $36.1 \%$ higher than the cyclostationary feature detection method. [8] have proposed that duty cycle (DC) and channel occupancy (COR) measurements are used to characterize the availability of white space in cognitive radio systems (CRS). [9] studied the joint impact of RF impairments on the spectrum sensing of CR systems based on energy detection in a multichannel environment. In particular, assuming Rayleigh fading, the author provides novel closed-form expressions to evaluate the probability of detection and false alarms. [10] proposed an improved channel occupancy estimation (iCOR) method that uses a high false alarm probability to increase sensitivity without the overestimation usually associated with high false alarm probability. The electromagnetic spectrum analysis method based on the time-frequency domain can effectively improve the estimation of background noise. [11] proposed a random multipath model for receiving signals, in which both the transmitter and receiver have directional antennas and are located in the same rectangular room. In indoor positioning applications, it is necessary to eliminate the electromagnetic interference of household appliances, and the background noise estimation is more important. Some scholars considered that the existing spectrum sensing algorithms are difficult to apply to practical applications and lead to waste of channels and energy resources, and [12] proposed an energy-saving spectrum-sensing algorithm. This algorithm can maximize the energy utilization efficiency under the premise of detecting sufficient channel available time, which is of great significance for reducing the cost of network deployment.

In order to provide meaningful data on spectrum usage, the occupancy measurement describing the utilization of a specific frequency band should be performed in a specific area rather than a single location. [13] described a comprehensive method for measuring and analyzing spectrum occupancy. [14] proposed a spectrum scanning method based on Bayesian inference to estimate the channel occupancy. This method takes into account the false detection probability and detection probability of the spectrum sensor to make the estimation of channel occupancy more accurate. [8] analyzed the problem of estimating the main channel COR based on the spectrum sensing decision and derived a tight closed-form expression for the required sensing sample size. However, different background noise distributions will be generated in different environments, which will directly affect the accuracy of channel estimation. One of the important judgment conditions for spectrum occupancy is noise threshold estimation, also called background noise estimation. Electromagnetic spectrum database as an important means to describe the electromagnetic environment. [15] optimized the spectrum sensing based on the IEEE 1900.6 standard to support the spectrum database. Especially in a complex environment, background noise estimation will directly affect the reliability of the electromagnetic spectrum database. The accurate estimation of background noise is one of the important indicators to improve the accuracy of spectrum sensing. [16] combined time-frequency domain electromagnetic spectrum data to solve the problem of accurately modeling the spectrum occupancy patterns of real radio communication systems, which is an important aspect of cognitive radio (CR) network research. [17] studied the spatiotemporal opportunity detection problem of spectrum heterogeneous cognitive radio network. The author proposes that at a given time, secondary users (SU) in different locations may encounter different spectrum access opportunities. The estimation of the background noise envelope of the time-frequency domain spectrum will directly affect the occupancy of the target frequency band determined by the secondary user.

\section{Worked}

We focus on electromagnetic spectrum detection methods based on time-frequency domain energy detection data. The method is simple and applicable, and it has higher real-time performance and reliability. We propose a target frequency band background noise estimation based on the time-frequency domain electromagnetic spectrum detection method. It estimates the fluctuation range of background 
noise according to the maximum hold method and the minimum hold method and combines the forward difference algorithm to estimate the background noise envelope curve. Finally, it combines the collected electromagnetic spectrum data to analyze and estimate the background noise of the target frequency band.

\section{Electromagnetic Spectrum Detection Based on Time-Frequency Domain}

Coastal stations provide ships with daily public communication services, acting as a relay between users and ships, broadcasting navigation warnings, weather forecasts, and other maritime emergency and safety information for ships. Its operating frequency is assigned by the International Telecommunication Union (ITU), and the identification of coast stations consists of 9 decimal digits starting with 00 . The spectrum distribution consists of the energy of each frequency point, it is affected by free space propagation loss and special geographic environment, and the detected instantaneous electromagnetic spectrum data will fluctuate to a certain extent. It is necessary to preprocess the electromagnetic spectrum data in the time-frequency domain to accurately obtain the background noise of the target frequency band.

4.1. Energy Detection and Preprocessing. The collected radio frequency data undergoes fast Fourier transform (FFT) to obtain energy values at different frequency points. The energy detection method can be expressed as

$$
x(t)= \begin{cases}n(t), & H_{0}, \\ h(t) s(t)+n(t), & H_{1},\end{cases}
$$

where $H_{0}$ is defined as noise, and $H_{1}$ is defined as effective signal. $n(t)$ is noise data, $s(t)$ is the signal data, and $h(t)$ is the channel parameter. $t$ represents a certain frequency point. $x(t)$ represents the energy detection value at frequency $t$ after the target frequency band passes through FFT.

(1) represents the result of an energy detection in the target frequency band. The effective signal and background noise can be distinguished by the threshold setting method. When processing the energy distribution data of the target frequency band, many researchers will use the horizontal line threshold method to distinguish between noise and effective signal. The selection of the threshold directly affects the probability of false detection $\left(P_{f a}\right)$ and the probability of missed detection $\left(P_{m}\right)$ of spectrum sensing. It is assumed that the set threshold is $T$. When $T$ is slightly greater than $n(t)$, most of the noise can be accurately determined. However, $n(t)$ will fluctuate due to environmental factors, and there are differences in $n(t)$ in different frequency bands. As $T$ increases, the probability of a weak signal being judged as noise increases. As $T$ decreases, the probability of noise being judged as a valid signal increases (see Figure 1). Therefore, it sets an appropriate $T$ to ensure lower $P_{f a}$ and $P_{m}$, as shown in the shaded part in Figure 1. In this way, the detection probability of spectrum sensing can be improved.

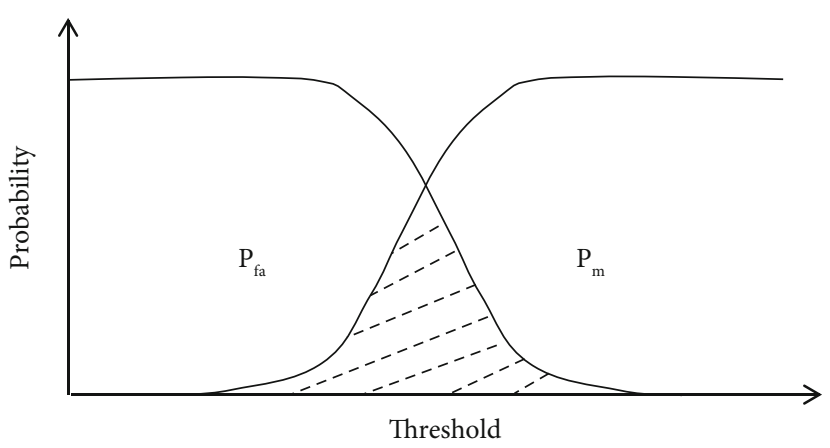

FIgURE 1: In the process of spectrum sensing, the relationship between $P_{f a}, P_{m}$, and the threshold setting.

In a complex environment, the fluctuation of the spectrum energy in the noise frequency band will be relatively large. This will result in a higher $P_{f a}$ in spectrum detection. In wireless communication systems, noise can be described by a normal distribution. Therefore, the fluctuation of noise can be smoothed by averaging the data of multiple frames of spectrum detection. It assumes that $n$ frames of timefrequency domain spectrum data are collected. It can be expressed as

$$
x_{\text {aver }}(t)=\frac{1}{n} \sum_{i=1}^{n} x(t) \text {, }
$$

where $i$ defines the spectrum data collected in the timefrequency domain spectrum of the $i$-th frame.

In order to calculate the background noise envelope of the target frequency band in the current environment, we propose to estimate the lower envelope of the background noise with the minimum hold method of the electromagnetic spectrum in the time-frequency domain and estimate the upper envelope of the background noise with the maximum hold method of the electromagnetic spectrum in the time-frequency domain. The upper envelope estimate of background noise can be expressed as

$$
x_{\max }(t)=\max (x(t)), t=1, \cdots, n .
$$

The lower envelope estimate of the background noise can be expressed as

$$
x_{\min }(t)=\min (x(t)), t=1, \cdots, n .
$$

If the signal existing in the target frequency band is a discontinuous signal, the minimum hold method is used to estimate the noise envelope. The maximum preservation method estimates the maximum threshold of noise fluctuations, which can avoid setting an excessively high noise threshold resulting in a higher $P_{m}$.

4.2. Background Noise Estimation. After it obtains the upper envelope and lower envelope of the background noise, the effective signal needs to be filtered out. The forward difference algorithm to estimate the adaptive threshold curve 


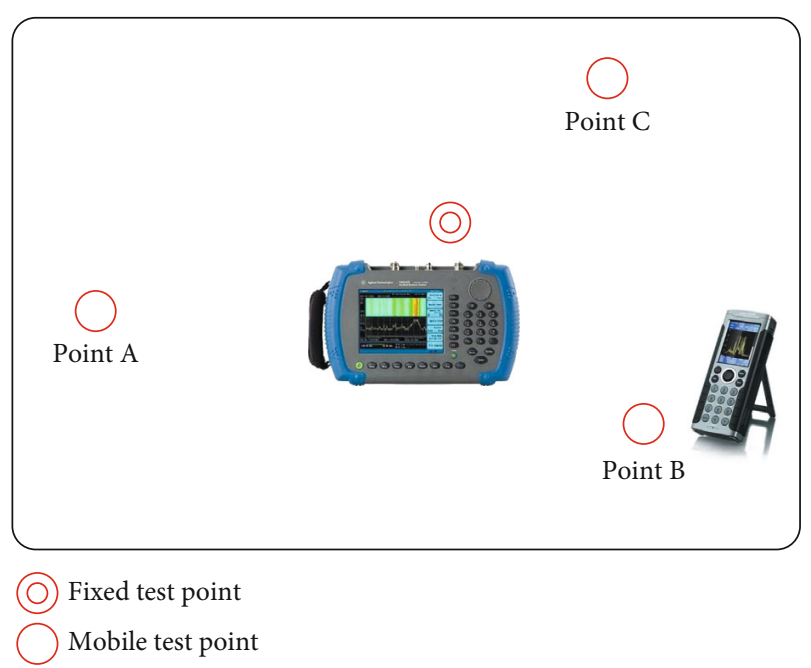

FIGURE 2: Schematic diagram of spectrum detection equipment and signal source location.

(ATC) is used to estimate the noise envelope [5]. The signal of the envelope is effectively filtered through the adjustment variable $D$. According to (2), calculating the average value of $N$ frames of data is smoother than the instantaneous frequency spectrum. In the instantaneous frequency spectrum, the effective signal can be seen as a pulse waveform. There are two slopes with larger absolute values before and after the center frequency. It assumes that the difference between adjacent spectral data is defined as $D_{v}$, the forward calculation of the spectral difference distribution between adjacent frequency points. It can be expressed as

$$
\mid \text { data }_{\mathrm{i}}-\text { data }\left._{i-1}\right|_{N}=D_{v} \text {. }
$$

It sets the difference threshold of the frequency spectrum, and after comparing and calculating the difference between adjacent frequency spectrums, it judges the front and back edge pulses of the signal. The spectral energy of the signal is filtered out, and the output is the noise curve. It can be expressed as

$$
n_{i}= \begin{cases}\text { data }_{i}, & D_{v} \leq D, \\ \text { data }_{i-1}, & D_{v}>D,\end{cases}
$$

where the frequency point is defined as $i$, the difference threshold is defined as $D$, and the output corresponding to each frequency point in the noise curve is defined as $n_{i}$.

Instantaneous spectrum data is difficult to improve the detection efficiency of effective signals. In order to effectively estimate the background noise, we propose a recognition and positioning method combined with effective signals to improve the detection probability of the spectrum. According to the time-frequency domain spectrum data, the gradient of the adjacent spectrum is calculated, and the rising edge and the falling edge boundary of the effective signal are obtained. It assumes that $r$ is the RSSI at the frequency

\begin{tabular}{|c|c|c|c|}
\hline $\begin{array}{l}\text { Frequency } \\
(\mathrm{kHz})\end{array}$ & Point A (dBm) & Point B $(\mathrm{dBm})$ & Point $\mathrm{C}(\mathrm{dBm})$ \\
\hline 2174.5 & -83.0 & -106.6 & -103.6 \\
\hline 2182 & -102.3 & -101.2 & -104.3 \\
\hline 2187.5 & -96.4 & -102.5 & -103.2 \\
\hline 4125 & -94.3 & -103.4 & -106.1 \\
\hline 4174 & -103.5 & -106.4 & -106.4 \\
\hline 4177.5 & -104.6 & -104.9 & -105.5 \\
\hline 4207.5 & -105.2 & -103.1 & -102.8 \\
\hline 6209 & -86.1 & -106.3 & -106.0 \\
\hline 6215 & -98.0 & -104.2 & -105.7 \\
\hline 6264.5 & -99.3 & -106.7 & -106.3 \\
\hline 6268 & -104.8 & -105.4 & -107.0 \\
\hline 6312 & -103.2 & -107.0 & -105.7 \\
\hline 8258 & -100.6 & -106.9 & -103.5 \\
\hline 8291 & -106.1 & -108.9 & -107.0 \\
\hline 8376.5 & -102.8 & -104.0 & -107.0 \\
\hline 8395 & -106.2 & -106.9 & -107.0 \\
\hline 8414.5 & -105.1 & -106.2 & -107.0 \\
\hline 12260 & -101.7 & -105.2 & -107.0 \\
\hline 12290 & -100.2 & -108.4 & -107.0 \\
\hline 12302 & -98.3 & -103.1 & -107.0 \\
\hline 12335 & -101.4 & -105.6 & -107.0 \\
\hline 12510.5 & -104.2 & -105.6 & -107.0 \\
\hline 12520 & -104.9 & -106.6 & -107.0 \\
\hline 12546.5 & -107.0 & -105.9 & -107.0 \\
\hline 12577 & -107.0 & -106.3 & -107.0 \\
\hline 16420 & -107.0 & -105.2 & -107.0 \\
\hline 16516 & -107.0 & -105.6 & -107.0 \\
\hline 16695 & -103.5 & -106.8 & -107.0 \\
\hline 16762 & -102.1 & -105.0 & -107.0 \\
\hline 16804.5 & -104.4 & -107.0 & -107.0 \\
\hline 18795 & -107.0 & -107.0 & -107.0 \\
\hline 18819 & -107.0 & -107.0 & -107.0 \\
\hline 22039 & -107.0 & -107.0 & -107.0 \\
\hline
\end{tabular}

TABLE 1: Measurement data of mobile monitoring points.

$f$ and time $t$, and the time-frequency domain energy matrix can be obtained. It can be expressed as

$$
N=\left[\begin{array}{ccc}
N_{0,0} & \cdots & N_{0, f-1} \\
\vdots & \ddots & \vdots \\
N_{t-1,0} & \cdots & N_{t-1, f-1}
\end{array}\right]
$$

There are generally two methods for gradient calculation, including horizontal gradient and vertical gradient. It can be expressed as

$$
G_{f, t}=\left\{\begin{array}{l}
N_{i+1, j}-N_{i, j} \\
N_{i, j+1}-N_{i, j}
\end{array}, i=0, \cdots, t j=0, \cdots, f .\right.
$$




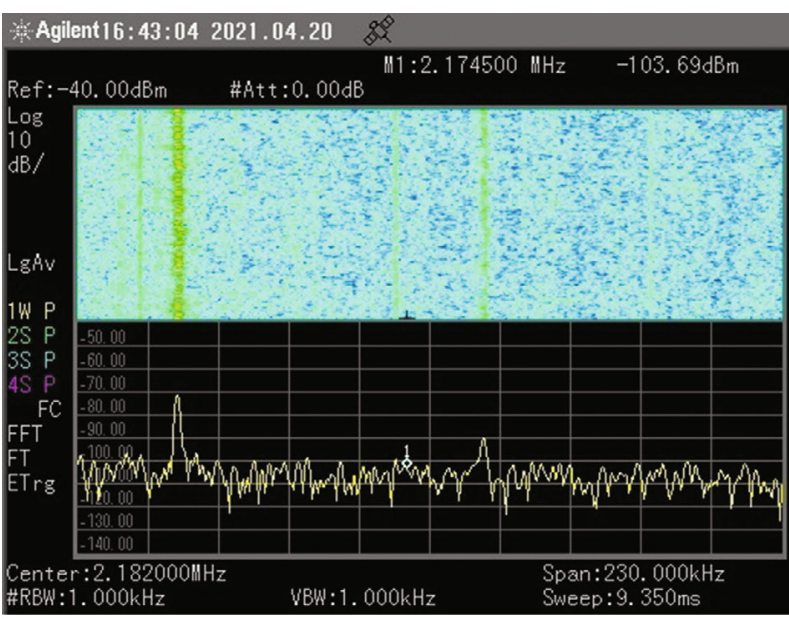

(a) Center frequency is $2.1745 \mathrm{MHz}$

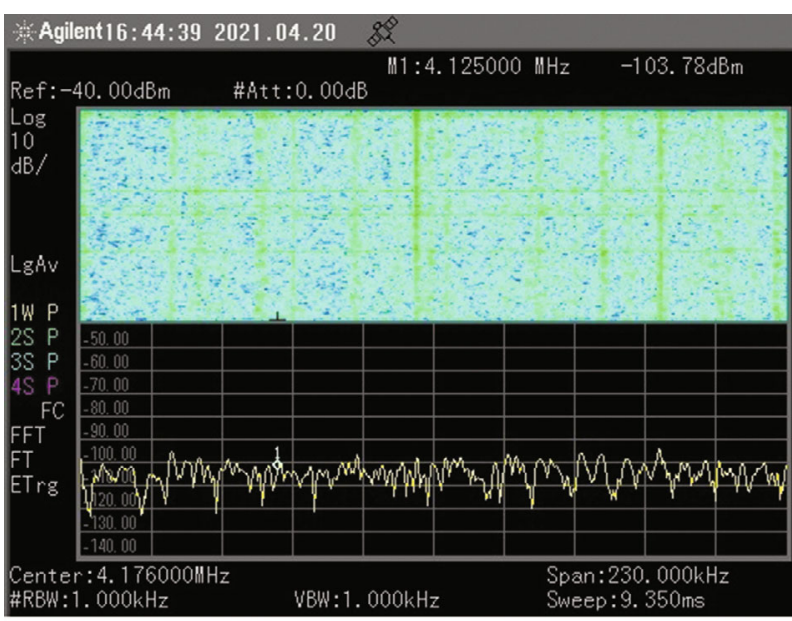

(b) Center frequency is $4.125 \mathrm{MHz}$

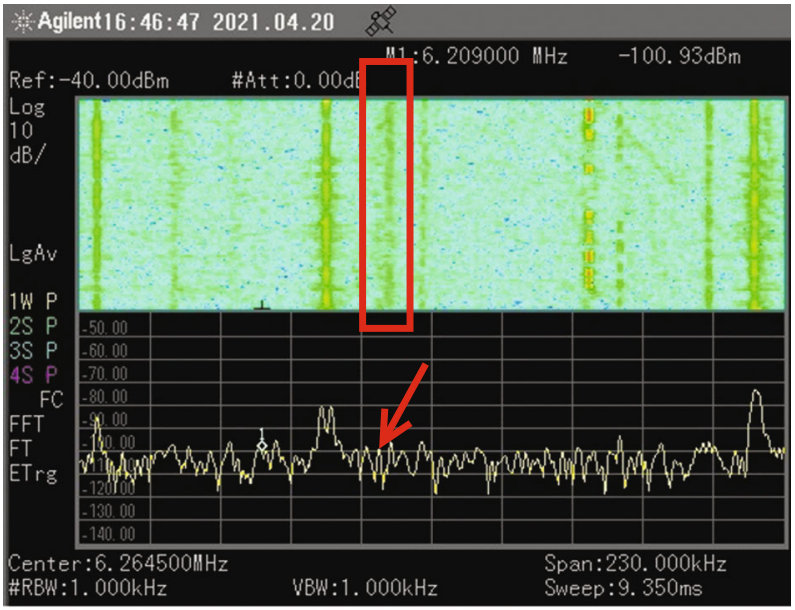

(c) Center frequency is $6.209 \mathrm{MHz}$

Figure 3: $2 \mathrm{MHz}-6 \mathrm{MHz}$ frequency band time-frequency domain spectrum (bandwidth is $230 \mathrm{kHz}$ ).

As time changes, the frequency spectrum of the signal is mainly distributed on a certain frequency point. Therefore, we use the transverse gradient to get the energy gradient matrix. It can be expressed as

$$
G_{f, t}=\left[\begin{array}{cccc}
N_{0,1}-N_{0,0} & \cdots & N_{0, t-1}-N_{0, t-2} & 0 \\
\vdots & \ddots & \vdots & \vdots \\
N_{t-1,1}-N_{t-1,0} & \cdots & N_{t-1, f-1}-N_{t-1, f-2} & 0
\end{array}\right] .
$$

Finally, it judges whether it is the upper edge or the lower edge of the valid signal according to the magnitude of the gradient value. The effective signal of the target frequency band can be marked, and the background noise area can be obtained at the same time.

\section{Detection and Analysis}

The coast station has 34 operating frequency points, which are distributed between the $2 \mathrm{MHz}$ and $23 \mathrm{MHz}$ frequency band. The test equipment are Agilent N9342C RF analyzer, portable handheld field strength meter, Beidou positioning terminal, notebook computer, and other related equipment. The conditions of the test site are as follows:

(1) In the field of 1 antennas, set up a fixed test point and continue testing

(2) In the construction project area, set up a total of 3 mobile test points $\mathrm{A} / \mathrm{B} / \mathrm{C}$, mainly to test the background noise in the temporary antenna area to be built (point $\mathrm{A} / \mathrm{B}$ ) and the short-distance construction area (point $\mathrm{C}$ ). Point $\mathrm{A} / \mathrm{B} / \mathrm{C}$ is the planned test location, and a suitable location nearby can be selected for testing according to the actual situation of the site

(3) It is finally determined according to the actual situation, and the fixed test point is set in the radio room (because it needs to be connected to the radio's short-wave antenna). There are 4 mobile test points (including inside the station). The test points are the top of the radio 


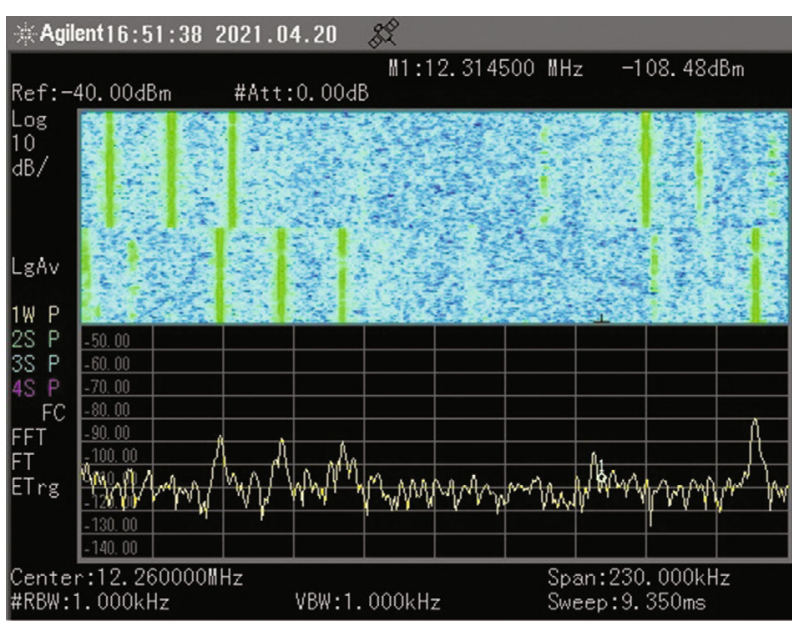

(a) Center frequency is $12.26 \mathrm{MHz}$

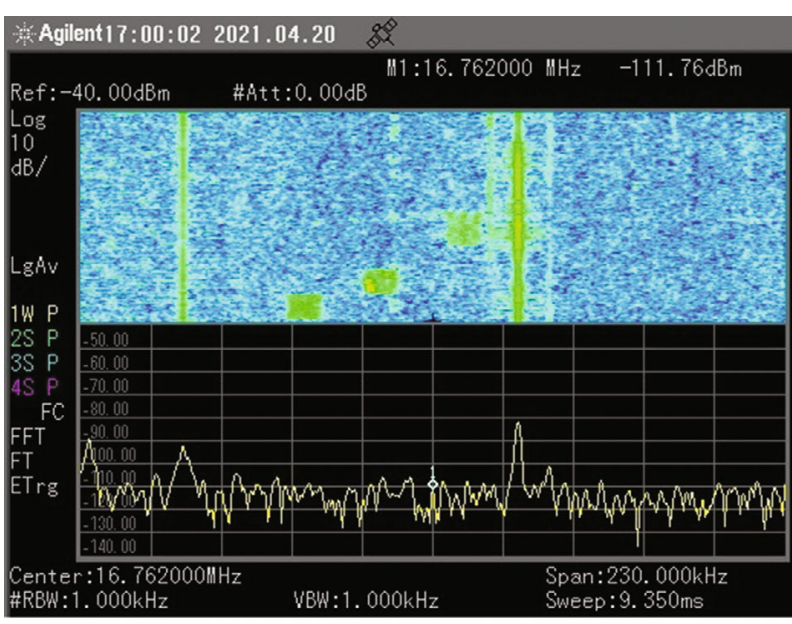

(b) Center frequency is $16.762 \mathrm{MHz}$

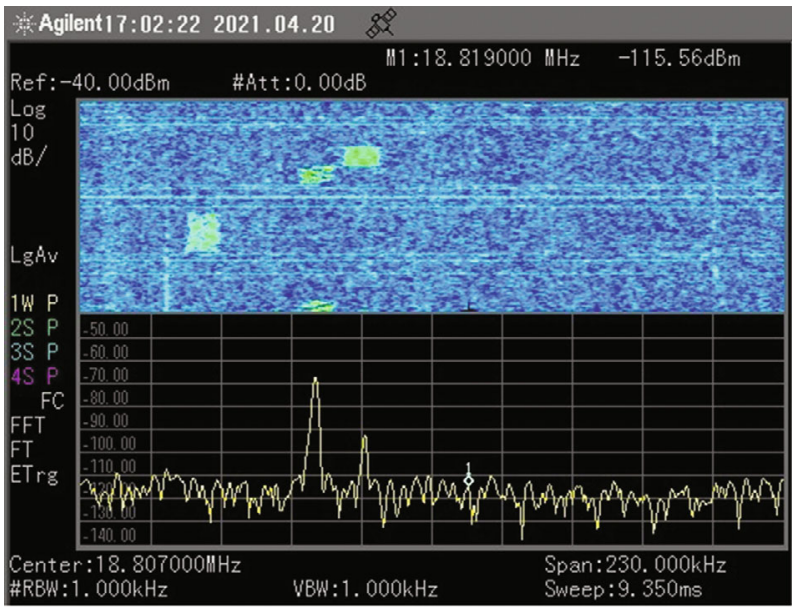

(c) Center frequency is $18.819 \mathrm{MHz}$

Figure 4: $12 \mathrm{MHz}-18 \mathrm{MHz}$ frequency band time-frequency domain spectrum (bandwidth is $230 \mathrm{kHz}$ ).

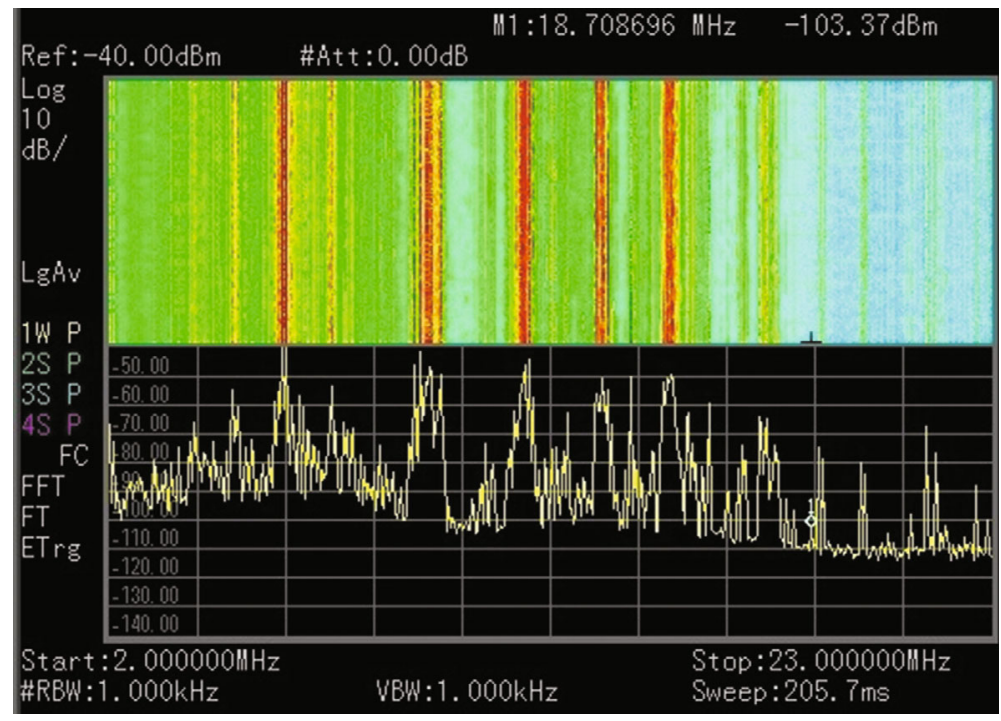

FIGURE 5: The time-frequency domain spectrum data of the $2 \mathrm{MHz}-23 \mathrm{MHz}$ frequency band acquired by the Agilent N9342C RF analyzer. 


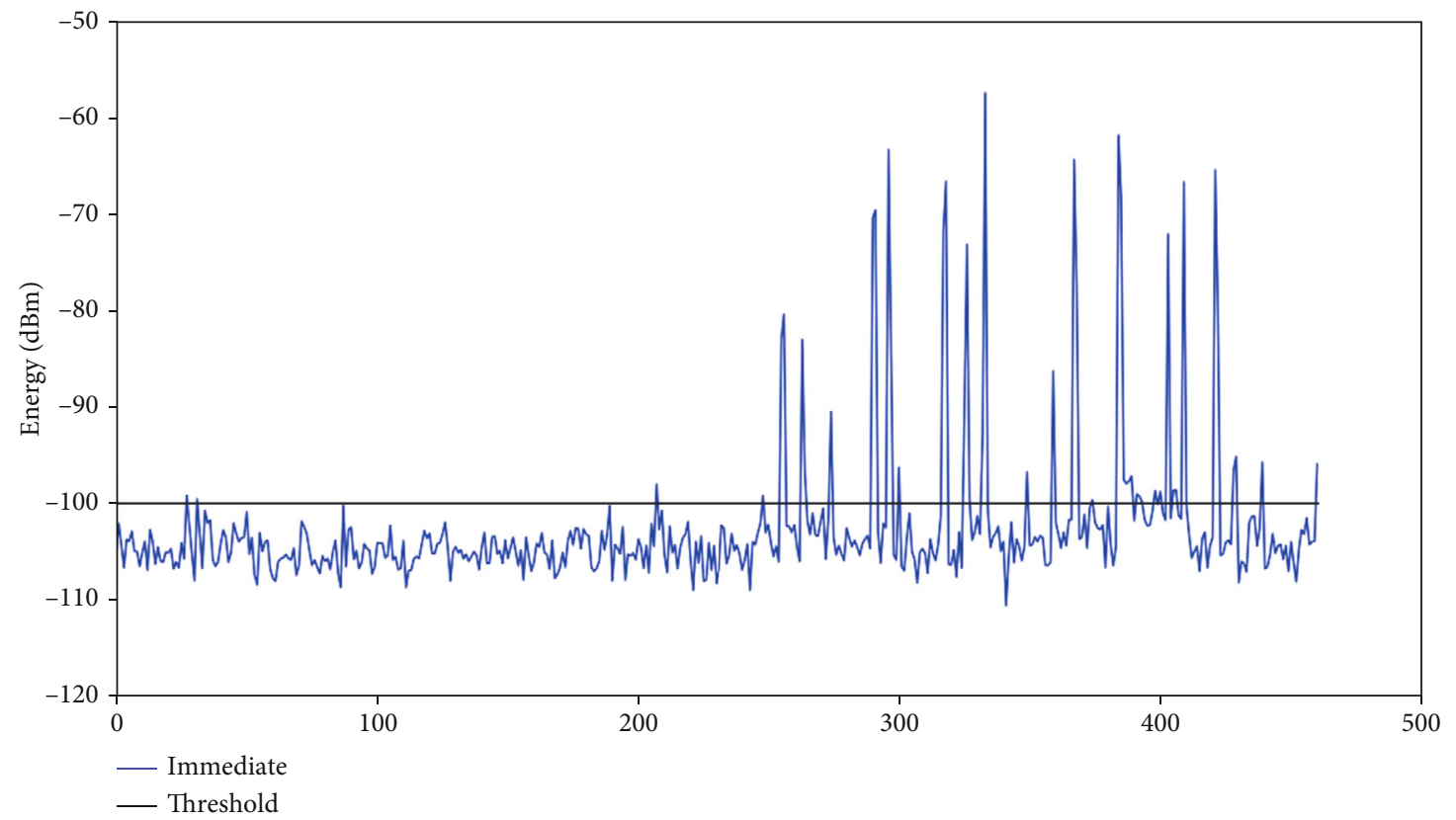

(a) $60 \mathrm{MHz}-110 \mathrm{MHz}$

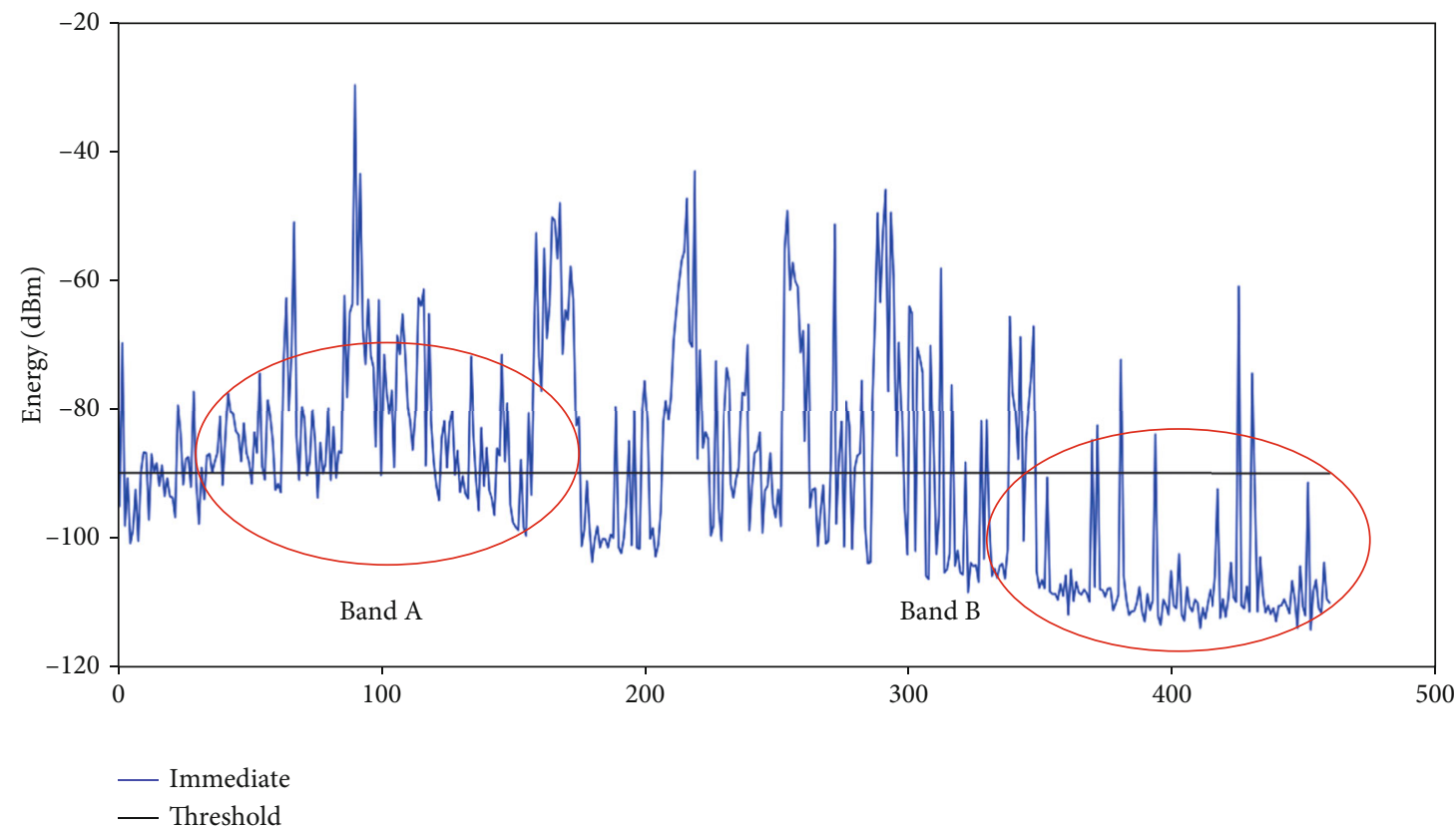

(b) $2 \mathrm{MHz}-23 \mathrm{MHz}$

FIgURE 6: Spectrum data of different frequency bands.

(4) Computer room, point A on the southwest side of the construction project, point $\mathrm{B}$ on the southeast side of the construction project, and point $\mathrm{C}$ on the northeast side of the construction project

At the mobile monitoring points, a handheld RF field strength meter is used for testing. Every four hours, a portable field strength meter is used to test various mobile monitoring points. The mobile measurement records the signal strength of each frequency point. The influence of background noise is determined according to different energy fluctuations of frequency points (see Figure 2). The test results are shown in Table 1 . The signal strength in the $2 \mathrm{MHz}-8 \mathrm{MHz}$ frequency band is $-90 \mathrm{dBm}$ to $-100 \mathrm{dBm}$, and the signal strength in the $12 \mathrm{MHz}-22 \mathrm{MHz}$ frequency band is $-100 \mathrm{dBm}$ to $-110 \mathrm{dBm}$. It is based on the test data analysis of the other three moving points, and the signal strength of the entire test frequency band is $-100 \mathrm{dBm}$ to $-110 \mathrm{dBm}$. The $4207.5 \mathrm{kHz}$ frequency of the mobile monitoring point $\mathrm{A}$ is selected. After comparing the test data, it can 


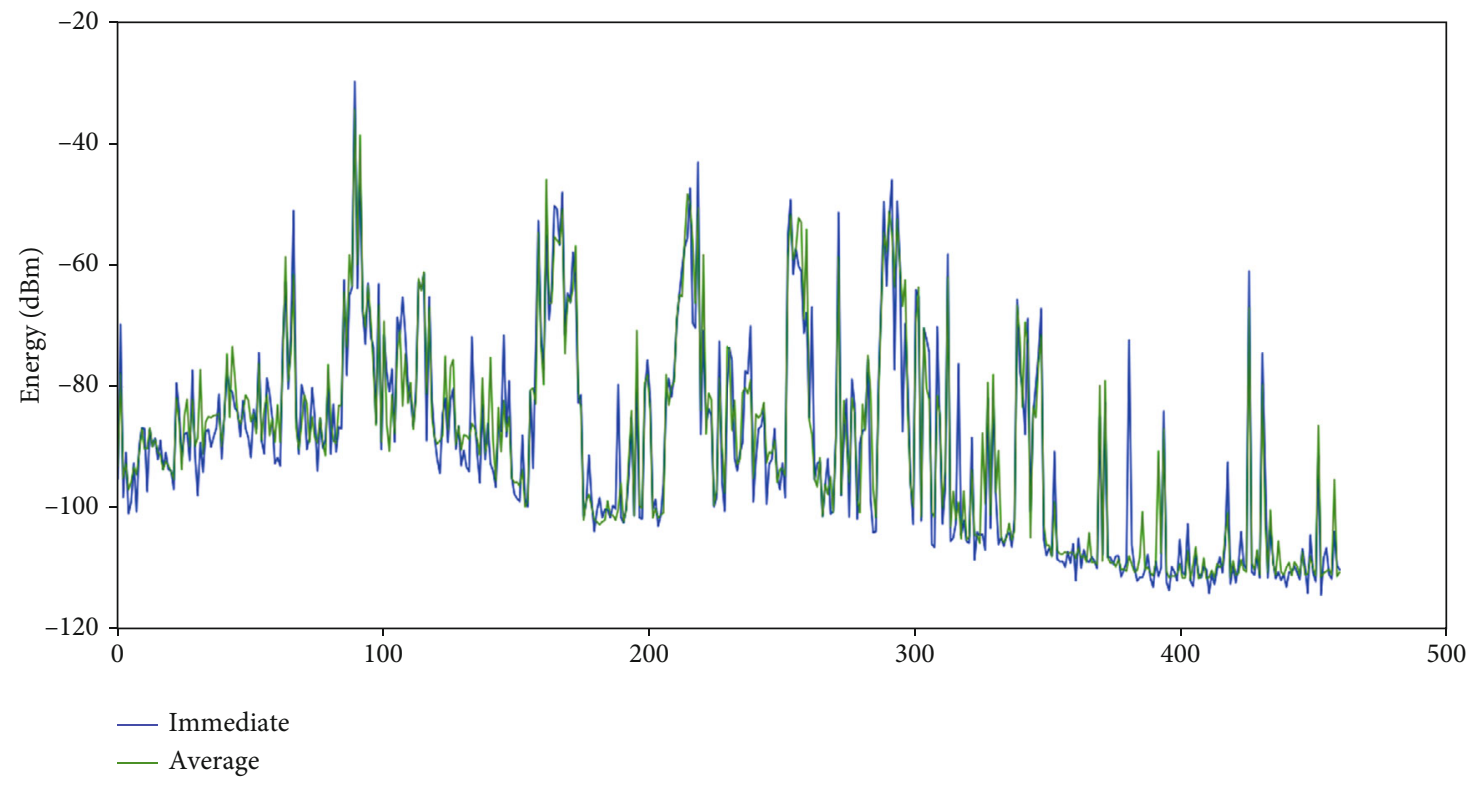

FIGURE 7: Instantaneous frequency spectrum and mean frequency spectrum.

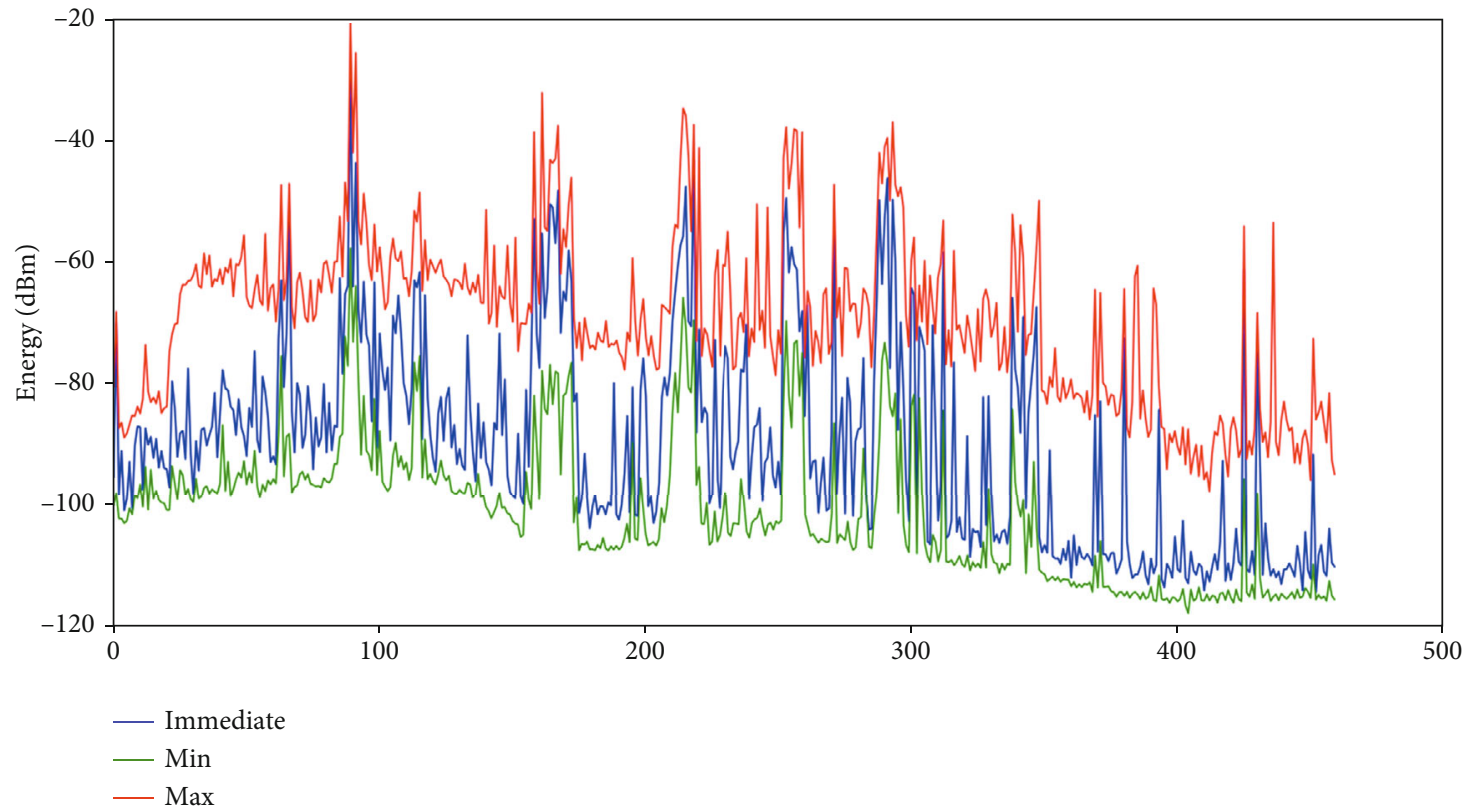

FIgURE 8: The upper envelope and lower envelope of the background noise.

be preliminarily determined that the background noise is about $-95 \mathrm{dBm}(2 \mathrm{MHz}-8 \mathrm{MHz}$ frequency band) and $-105 \mathrm{dBm}(12 \mathrm{MHz}-22 \mathrm{MHz}$ frequency band). We collected the time-frequency domain spectrum with center frequency of $2.1745 \mathrm{MHz}, 4.125 \mathrm{MHz}, 6.209 \mathrm{MHz}, 12.26 \mathrm{MHz}$, $16.762 \mathrm{MHz}$, and $18.819 \mathrm{MHz}$, the sampling bandwidth is $230 \mathrm{kHz}$, and the sampling interval is $0.5 \mathrm{kHz}$, to ensure that it can cover all the signal frequency points in Table 1.

The background noise of the $2.1745 \mathrm{MHz}, 4.125 \mathrm{MHz}$, and $6.209 \mathrm{MHz}$ frequency bands is about $-100 \mathrm{dBm}$ (see Figure 3). The background noise envelope in the frequency band does not fluctuate much. The frequency point position of the effective signal can also be clearly determined from the time-frequency domain spectrum. The linear threshold can be used to quickly determine the noise and the effective signal. The background noise of the $12.26 \mathrm{MHz}, 16.762 \mathrm{MHz}$, and $18.819 \mathrm{MHz}$ frequency bands is about $-105 \mathrm{dBm}$ (see Figure 4). Compared to the $2 \mathrm{MHz}-6 \mathrm{MHz}$ frequency band, the overall background noise of this frequency band is reduced by $5 \mathrm{~dB}$. If the same decision threshold $T$ is used, the probability of missed detection of weak signals in this frequency band is higher, which will ultimately affect the 


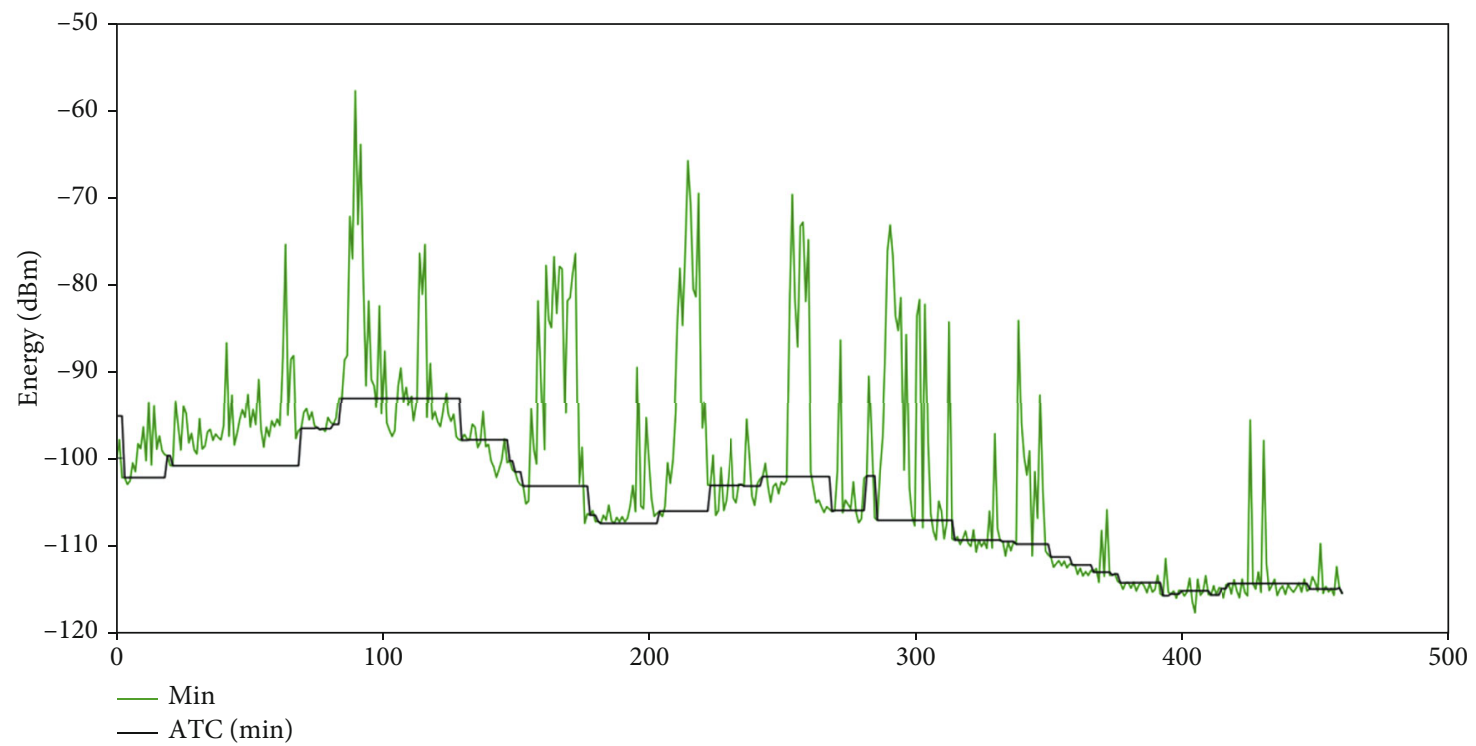

Figure 9: The upper envelope and lower envelope of the background noise.

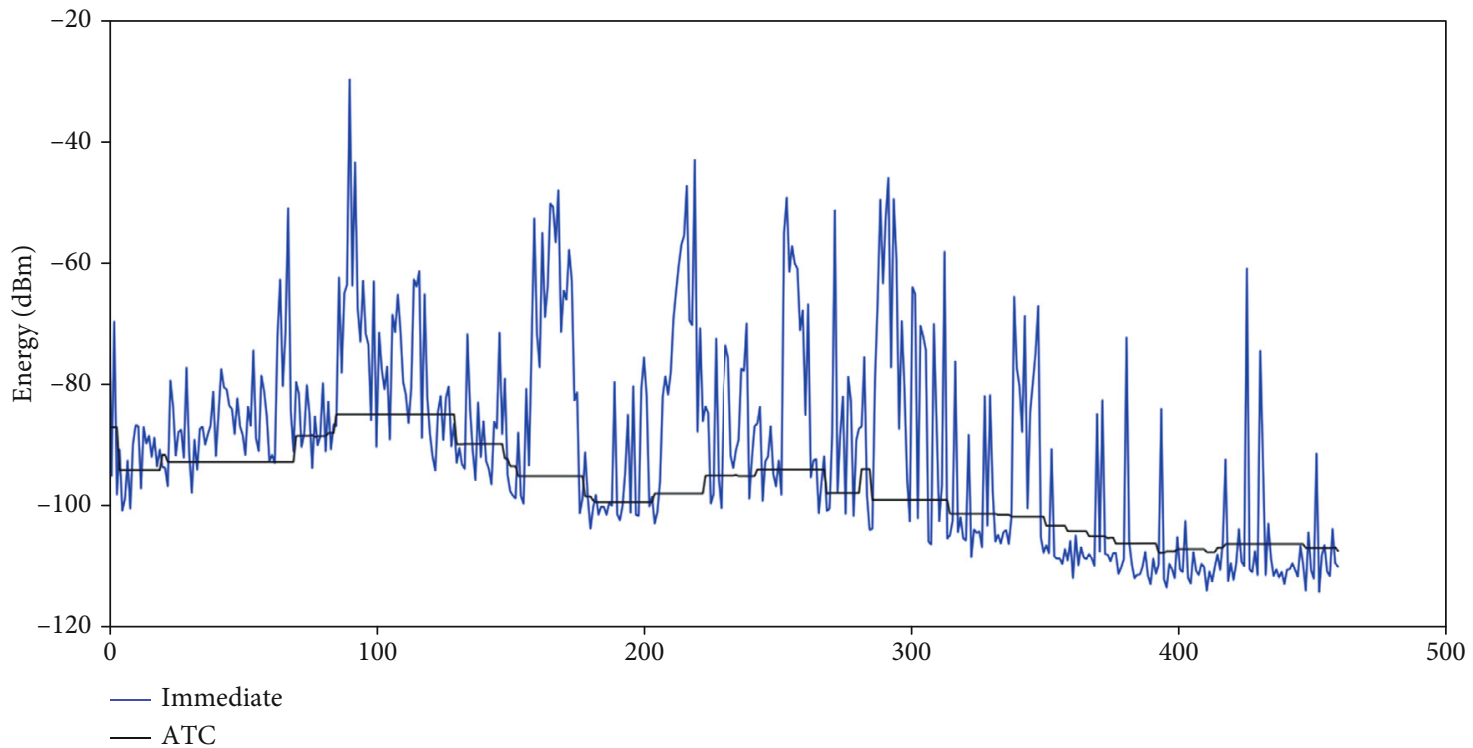

FIGURE 10: The upper envelope and lower envelope of the background noise.

overall detection probability. The time-frequency domain spectrum is also called waterfall chart. The horizontal axis represents frequency, the vertical axis represents time, and the color in the figure represents the received signal strength (received signal strength indication). The darker the color (red), the stronger the RSSI, and the lighter the color (blue), the weaker the RSSI. Comparing the RSSI in the $2 \mathrm{MHz}-$ $6 \mathrm{MHz}$ frequency band and the $12 \mathrm{MHz}-18 \mathrm{MHz}$ frequency band, the overall background noise energy of the lower frequency band is higher. For low-frequency bands, the energy of background noise fluctuates over time, which will bury weak signals in the noise and increase the probability of missed detection. It is difficult to identify the effective signal through the instantaneous frequency spectrum, as shown in the position of the red arrow in Figure 3(c). However, through the distribution of the time-frequency domain spectrogram, it can be clearly found that there is a signal at the channel, as shown in the position of the red box in Figure 3(c). In the higher frequency band, the background noise energy is lower, so it is easier to judge the weak signal. However, a fixed energy threshold cannot be set, because it is not suitable for signal detection in the full frequency spectrum.

At fixed monitoring points, Agilent spectrum analyzers are used to collect electromagnetic spectrum data in the time-frequency domain. The parameters of the spectrum analyzer are set as follows: in order to cover all frequency points, the span SPAN is set to $230 \mathrm{kHz}$, the resolution bandwidth (RBW) is $1 \mathrm{kHz}$, the view bandwidth (VBW) is $1 \mathrm{kHz}$, the reference level is $-40 \mathrm{dBm}$, the attenuation is $0 \mathrm{~dB}$, and the sampling interval is $100 \mathrm{~ms}$. The collected 


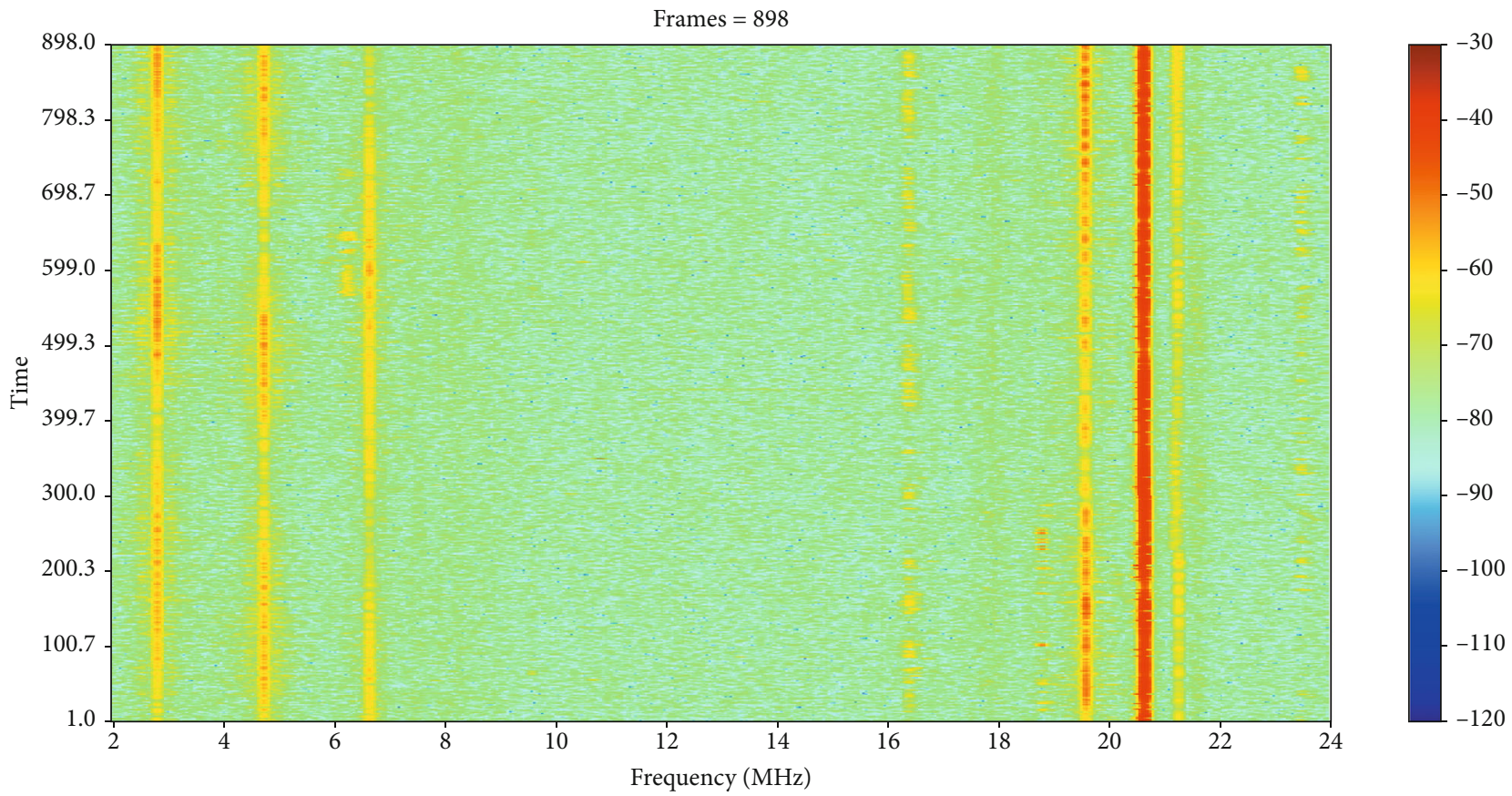

(a) Time-frequency domain spectrum data

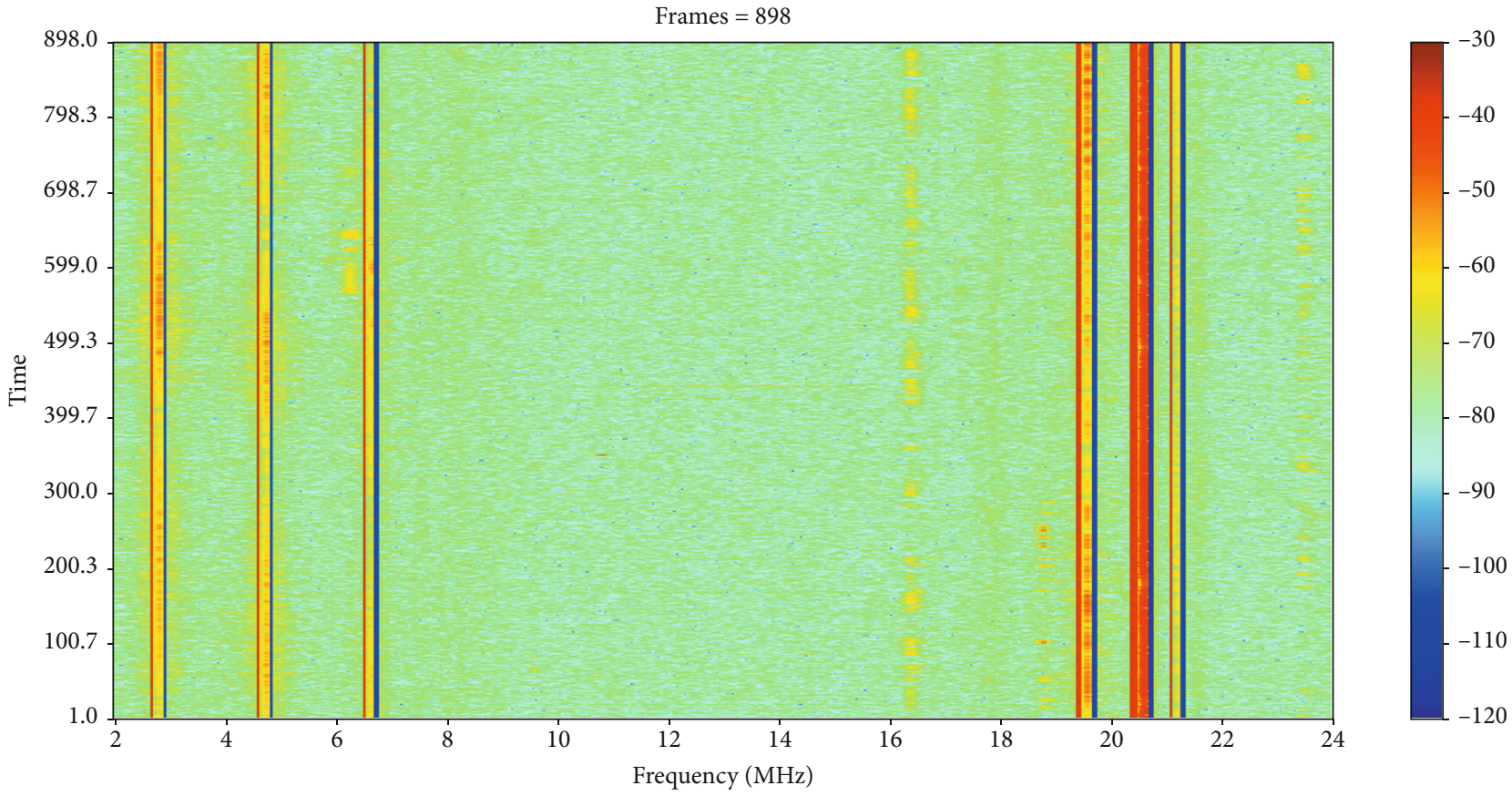

(b) Effective signal for energy gradient estimation

Figure 11: Time-frequency domain spectral energy gradient and effective signal estimation.

spectrum data is saved in a CSV file with a size of about $8 \mathrm{MB}$ as 1500 frames of data. The time-frequency domain wide-band scanning range of the spectrum analyzer is $2 \mathrm{MHz}-23 \mathrm{MHz}$, and the result is shown in Figure 5. In the lower frequency band and the middle frequency band, the signal reception strength is strong, and multiple signal pulses can be clearly observed. The signal surroundings are accompanied by higher noise energy, and the noise floor is about
$-80 \mathrm{dBm}$ to $-90 \mathrm{dBm}$. The noise floor in the higher frequency band is about $-100 \mathrm{dBm}$ to $-110 \mathrm{dBm}$.

The instantaneous frequency spectrum of a certain frequency band of HF and VHF is shown in Figure 6. The background noise of the VHF spectrum is relatively flat compared to the HF spectrum. For the VHF spectrum, assuming $T=-100 \mathrm{dBm}$, most of the noise can be judged, as shown in Figure 6(a). For the high-frequency spectrum, 
suppose $T=-90 \mathrm{dBm}$, as shown in Figure 6(b). Then, the noise of band $\mathrm{A}$ is judged to be a valid signal, and $P_{f a}$ is higher. Weak signals in band $B$ are all judged to be noise, and $P_{m}$ is higher. The traditional linear threshold setting method is not suitable for wide-band spectrum scanning tasks due to its own characteristics. Moreover, the value of the threshold requires a large amount of a priori spectrum detection data to determine, and the actual application process is relatively tedious and time-consuming and laborious.

Because the mobile monitoring node has poor timeliness when measuring the instantaneous spectrum, it cannot accurately analyze the electromagnetic spectrum environment. Therefore, it is necessary to analyze the background noise of the target frequency band based on the electromagnetic spectrum data in the time-frequency domain. In order to reduce the influence of noise fluctuations, it performs average smoothing processing on multiframe detection data according to (2). The data after smoothing is shown in Figure 7.

It is necessary to reduce the energy fluctuation changes in the noise frequency band. Then calculate the upper envelope and lower envelope of the background noise according to (3) and (4) (see Figure 8). The envelope under background noise can basically fit the background noise curve of the instantaneous spectrum. The envelope on the background noise can be used as the maximum threshold for the background noise threshold setting. For different frequency bands, the background noise envelope estimation based on the time-frequency domain electromagnetic spectrum can dynamically estimate the noise floor.

According to (5) and (6), the effective signal of the lower envelope under the background noise is filtered out (see Figure 9). Among them, the parameter of $D$ is set to $0.282 \mathrm{dBm}$.

At this time, it adjusts the background noise according to the maximum threshold of the upper envelope and finally gets the background noise envelope curve in the target frequency band. Among them, the background noise envelope is increased by $8 \mathrm{~dB}$. The obtained background noise envelope curve can describe the noise floor of different frequency bands (see Figure 10).

As for the signal reception, it is better at night than during the day. From the perspective of noise floor, there is a difference of about $20 \mathrm{~dB}$ between the noise floor tested during the day and the noise floor tested at night. The reception situation of fixed monitoring points is better than that of mobile monitoring points, and the reception situation is worse near the construction test points, which can be known from the data received by different monitoring nodes. For spectrum data in the time-frequency domain, the background noise estimation of the instantaneous spectrum has certain limitations. It combines the time-frequency domain energy gradient matrix, and the effective signal of the target frequency band can be estimated quickly and effectively. According to (9) and the signal edge estimation method, the result of the effective signal estimation of the time-frequency domain spectrum is obtained (see Figure 11). Figure 11(a) shows the time-frequency domain spectrum data of a frequency band. The upper edge of the valid signal is marked with a red line, and the lower edge is marked with a blue line, as shown in Figure 11(b).
Although the energy gradient estimation method can estimate the effective signal in combination with the timefrequency domain spectrum data, there is still a certain probability of missed detection for the detection of discontinuous signals. This is also a problem that will be emphatically solved in the follow-up research.

\section{Conclusions}

Detecting and estimating the background noise of the electromagnetic spectrum environment of coast stations play an important role in measuring the quality of wireless communication in this area. We propose to estimate the upper envelope and lower envelope of the background noise by the maximum hold method and the minimum hold method, respectively. Combined with the forward difference algorithm, the background noise envelope curve is estimated. After the time-frequency domain electromagnetic spectrum data is processed, the noise floor of different target frequency bands is estimated, and the communication quality of the coast station is judged. This method combined with the time-frequency domain energy gradient estimation, the effective signal of the target frequency band can be quickly estimated, and according to the estimation of the background noise envelope and the effective signal, the background noise based on the time-frequency domain spectrum can be accurately determined. In the later period, we will study radio interference source detection and identification methods based on time-frequency domain data.

\section{Data Availability}

The data involved in this article are all obtained through the equipment Agilent N9342C RF analyzer.

\section{Conflicts of Interest}

The authors declare that they have no conflicts of interest.

\section{Acknowledgments}

The authors would like to thank the research was sponsored by the National Natural Science Foundation of China (62101166) and the Innovative Research Projects for Graduate Students in Ordinary Colleges and Universities in Hainan Province (Hys2020-216).

\section{References}

[1] M. López-Benítez and F. Casadevall, "Improved energy detection spectrum sensing for cognitive radio," IET Communications, vol. 6, no. 8, pp. 785-796, 2012.

[2] K. Umebayashi, K. Hayashi, and J. J. Lehtomäki, "Thresholdsetting for spectrum sensing based on statistical information," IEEE Communications Letters, vol. 21, no. 7, pp. 1585-1588, 2017.

[3] J. J. Lehtomäki, R. Vuohtoniemi, and K. Umebayashi, "On the measurement of duty cycle and channel occupancy rate," IEEE Journal on Selected Areas in Communications, vol. 31, no. 11, pp. 2555-2565, 2013. 
[4] Q.-T. Vien, H. X. Nguyen, R. Trestian, P. Shah, and O. Gemikonakli, "A hybrid double-threshold based cooperative spectrum sensing over fading channels," IEEE Transactions on Wireless Communications, vol. 15, no. 3, pp. 1821-1834, 2016.

[5] Z. Chen and Y. Zhang, "Cooperative energy detection algorithm based on background noise and direction finding error," $A E U$ - International Journal of Electronics and Communications, vol. 95, pp. 326-341, 2018.

[6] M. Hajiabadi, H. Khoshbin, and G. Abed Hodtani, "Cooperative spectrum estimation over large-scale cognitive radio networks," IET Signal Processing, vol. 11, no. 8, pp. 1006-1014, 2017.

[7] A. Bagwari and G. S. Tomar, "Multiple energy detection vs cyclostationary feature detection spectrum sensing technique," in 2014 Fourth International Conference on Communication Systems and Network Technologies, pp. 178-181, Bhopal, India, 2014.

[8] M. Lopez-Benitez and J. Lehtomaki, "On the sensing sample size for the estimation of primary channel occupancy rate in cognitive radio," in IEEE Wireless Conference and Networking Conference (WCNC 2016), Doha, Qatar, 2016.

[9] A.-A. A. Boulogeorgos, N. D. Chatzidiamantis, and G. K. Karagiannidis, "Energy detection spectrum sensing under RF imperfections," IEEE Transactions on Communications, vol. 64, no. 7, pp. 2754-2766, 2016.

[10] J. J. Lehtomaki, M. Lopez-Benitez, K. Umebayashi, and M. Juntti, "Improved channel occupancy rate estimation," IEEE Transactions on Communications, vol. 63, no. 3, pp. 643-654, 2015.

[11] T. Pedersen, "Stochastic multipath model for the in-room radio channel based on room electromagnetics," IEEE Transactions on Antennas and Propagation, vol. 67, no. 4, pp. 2591-2603, 2019.

[12] E. Pei, L. Li, W. Cheng, and J. Jiang, "An energy efficient spectrum sensing algorithm in EH-HCRSN," IEEE Access, vol. 6, pp. 63021-63032, 2018.

[13] M. Höyhtyä, A. Mämmelä, M. Eskola et al., "Spectrum occupancy measurements: a survey and use of interference maps," IEEE Communications Surveys \& Tutorials, vol. 18, no. 4, pp. 2386-2414, 2016.

[14] M. Riahi Manesh, S. Subramaniam, H. Reyes, and N. Kaabouch, "Real-time spectrum occupancy monitoring using a probabilistic model," Computer Networks, vol. 124, pp. 87-96, 2017.

[15] O. Holland, B. Bochow, and K. Katzis, "IEEE 1900.6b sensing support for spectrum databases," in 2015 IEEE Conference on Standards for Communication and Networking(CSCN), pp. 199-205, Tokyo, Japan, 2015.

[16] M. López-Benítez and F. Casadevall, “Time-dimension models of spectrum usage for the analysis, design, and simulation of cognitive radio networks," IEEE Transactions on Vehicular Technology, vol. 62, no. 5, pp. 2091-2104, 2013.

[17] Q. Wu, G. Ding, J. Wang, and Y.-D. Yao, "Spatial-temporal opportunity detection for spectrum-heterogeneous cognitive radio networks: two-dimensional sensing," IEEE Transactions on Wireless Communications, vol. 12, no. 2, pp. 516-526, 2013. 